\title{
Secondhand Smoke Exposure History Not Available
}

National Cancer Institute

\section{Source}

National Cancer Institute. Secondhand Smoke Exposure History Not Available. NCI

Thesaurus. Code C156839.

The history of secondhand smoke exposure is not available. 